\title{
Peripheral blood metabolome predicts mood change-related activity in mouse model of bipolar disorder
}

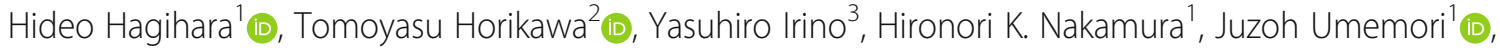 \\ Hirotaka Shoji ${ }^{1}$, Masaru Yoshida ${ }^{4,5}$, Yukiyasu Kamitani ${ }^{2,6}$ (ID and Tsuyoshi Miyakawa ${ }^{1 *}$ (D)
}

\begin{abstract}
Bipolar disorder is a major mental illness characterized by severe swings in mood and activity levels which occur with variable amplitude and frequency. Attempts have been made to identify mood states and biological features associated with mood changes to compensate for current clinical diagnosis, which is mainly based on patients' subjective reports. Here, we used infradian (a cycle $>24 \mathrm{~h}$ ) cyclic locomotor activity in a mouse model useful for the study of bipolar disorder as a proxy for mood changes. We show that metabolome patterns in peripheral blood could retrospectively predict the locomotor activity levels. We longitudinally monitored locomotor activity in the home cage, and subsequently collected peripheral blood and performed metabolomic analyses. We then constructed cross-validated linear regression models based on blood metabolome patterns to predict locomotor activity levels of individual mice. Our analysis revealed a significant correlation between actual and predicted activity levels, indicative of successful predictions. Pathway analysis of metabolites used for successful predictions showed enrichment in mitochondria metabolism-related terms, such as "Warburg effect" and "citric acid cycle." In addition, we found that peripheral blood metabolome patterns predicted expression levels of genes implicated in bipolar disorder in the hippocampus, a brain region responsible for mood regulation, suggesting that the brainperiphery axis is related to mood-change-associated behaviors. Our results may serve as a basis for predicting individual mood states through blood metabolomics in bipolar disorder and other mood disorders and may provide potential insight into systemic metabolic activity in relation to mood changes.
\end{abstract}

\section{Introduction}

Mood naturally changes over time with variable amplitude and frequency, sometimes in an infradian (a cycle $>24 \mathrm{~h}$ ) manner $[1,2]$. This is often accompanied by changes in physical activity [3]. In bipolar disorder, unusual swings in mood and activity levels from depressive to manic states are core phenomenological and clinical features. Such exaggerated fluctuations in mood and the related activity can cause significant distress and/or social and occupational impairment, often leading to high direct and indirect health care costs $[4,5]$. Currently, the clinical assessment and management of these conditions mainly

\footnotetext{
* Correspondence: miyakawa@fujita-hu.ac.jp

${ }^{1}$ Division of Systems Medical Science, Institute for Comprehensive Medical Science, Fujita Health University, Kutsukake-cho, Toyoake, Aichi 470-1192, Japan

Full list of author information is available at the end of the article
}

rely on clinicians' interview and patients' subjective description. To overcome the potential subjective biases, developing objective and quantitative measures is expected to compensate for the current procedures. To this end, substantial efforts have been invested into predicting mood states of patients with bipolar disorder based on biological features, such as heartbeat [6], spontaneous speech [7], and neural activity measured with functional magnetic resonance imaging [8]. Meanwhile, molecular omics approaches, such as metabolomics and proteomics, with which hundreds of molecules can be targeted simultaneously, are being applied to gain more complete profiles of a wide range of biological conditions and diseases $[9,10]$. However, such molecular omics-based approaches have not been well examined to predict mood states. While mood disorders are considered to be brain

(c) The Author(s). 2019 Open Access This article is distributed under the terms of the Creative Commons Attribution 4.0 International License (http://creativecommons.org/licenses/by/4.0/), which permits unrestricted use, distribution, and 
diseases, the use of peripheral samples has been desirable for clinical purposes, as the opportunity to obtain human brain tissues is limited [11].

Previous studies have attempted to detect biomarkers for mood disorders in the blood by comparing the metabolomes of patients to control subjects [11-13]. It is still not well understood whether or to what extent metabolomic alterations in peripheral blood are related to the shift of mood states. This may, in part, be due to the lack of animal models that exhibit behavioral phenotypes associated with infradian changes in mood states. We previously found that mice with the heterozygous knockout of the alpha-isoform of calcium/calmodulin-dependent protein kinase II (Camk2 $a^{+/-}$mice) exhibit various dysregulated behaviors, including exaggerated cyclic mood-change-associated activity in an infradian manner, in which locomotor activity (LA) spontaneously and recurrently increases and decreases in a period of approximately 10-20 days [14, 15]. Importantly, changes in the LA were correlated with changes in depression-like and anxiety-like behaviors [14], suggesting that $\operatorname{Camk} 2 a^{+/-}$mice are useful as an animal model which shows similar infradian oscillations of mood to those found in patients with bipolar disorder. CAMK2A is one gene suggested to be a candidate for bipolar disorder [16-18], and decreased CAMK2A mRNA has been found in the prefrontal cortex of patients with the disorder [19]. Camk $2 a^{+/-}$mice also harbor brain endophenotypes relevant to bipolar disorder, such as neuronal hyperexcitability [20] and immaturity $[15,21-23]$ in the hippocampus and decreased brain $\mathrm{pH}$ [24]. These findings suggest that Camk $2 a^{+/-}$mice provide a model for bipolar disorder which has good face and construct validity [25].

Using the Camk $2 a^{+/-}$mice, we previously showed that gene expression patterns in the hippocampus, a brain region implicated in mood regulation, could retrospectively predict the LA level of individual mice by using statistical learning algorithm [14]. We used cross-validated multivariate regression models to ensure the generalization ability of our prediction models. Generalization ability is the capacity to predict unseen samples, and ensuring and improving the generalization ability of models are believed to be crucial for practical use, including identification of subpopulations among patients with mood disorders [26]. In the present study, using this strategy, we sought to determine whether metabolome patterns in peripheral blood can predict individual LA levels of the mice, which would have potential for future translational applications. We also investigated whether gene expression levels in the brain can be predicted by peripheral blood metabolome patterns, aiming to gain insights into the link between systemic metabolic pathways and the regulation of brain gene expression in relation to the infradian mood changes.

\section{Materials and methods \\ Animals}

Thirty-seven adult ( $>8$ weeks old) male Camk $2 a^{+/-}$mice were used [14, 15, 27]. Every effort was made to minimize the number of animals used.

\section{Locomotor activity monitoring and blood sampling}

The LA data of Camk2a $a^{+/-}$mice analyzed in this study were the same as was used in a previous study [14]. Mice were singly housed with a $12 \mathrm{~h}$ light/dark cycle (lights on at 7:00 a.m.) and access to food and water ad libitum. The monitoring of LA and blood sampling was performed as previously described [14]. Briefly, LA was monitored for 72-82 days through a system that automatically analyzes the distance traveled by a mouse in its home cage [15]. Peripheral blood and brain tissue [14], was collected at zeitgeber time (ZT) 6-7 (ZT0, lights on; ZT12, lights off) from mice with short or long distances traveled (assessed by distance traveled during the $24 \mathrm{~h}$ before ZT0 on the sampling day; Fig. 1a, b). In this way, mice were selected for the sampling such that their $24 \mathrm{~h}$ LA levels varied among the 37 mice (Fig. 1c). The $24 \mathrm{~h} \mathrm{LA}$ was defined as distance traveled during the $24 \mathrm{~h}$ between ZT0 on the day before the sampling and ZT0 on the day of sampling. The $3 \mathrm{~h} \mathrm{LA}$ was defined as distance traveled in every $3 \mathrm{~h}$ window before sampling (ZT6) (Fig. 1b).

Mice were removed from the home cage, and immediately euthanatized by cervical dislocation. Blood was collected from the neck in tubes with $\mathrm{Na}$-heparin and centrifuged $\left(2200 \times g\right.$ at $4{ }^{\circ} \mathrm{C}$ for $\left.10 \mathrm{~min}\right)$ to prepare plasma. Plasma samples were stored at $-80^{\circ} \mathrm{C}$ until use.

\section{Gas chromatography mass spectrometry (GC/MS) analysis of blood samples}

GC/MS analysis was performed as described previously [28] using a GCMS-QP2010 Ultra (Shimazu, Kyoto, Japan) with a fused silica capillary column (CP-SIL 8 CB low bleed/MS; $30 \mathrm{~m} \times 0.25 \mathrm{~mm}$ inner diameter, film thickness $0.25 \mu \mathrm{m}$; Agilent Technologies, Palo Alto, CA, USA). Data processing was performed as described previously [28]. 2-isopropylmalic acid was used as an internal standard. Two mice whose metabolite contents could not be measured due to unknown reasons were excluded from further analyses. Hence, LA and metabolome data from 35 mutant mice were processed for correlation and prediction analyses (Additional file 2: Table S1), as described below. 


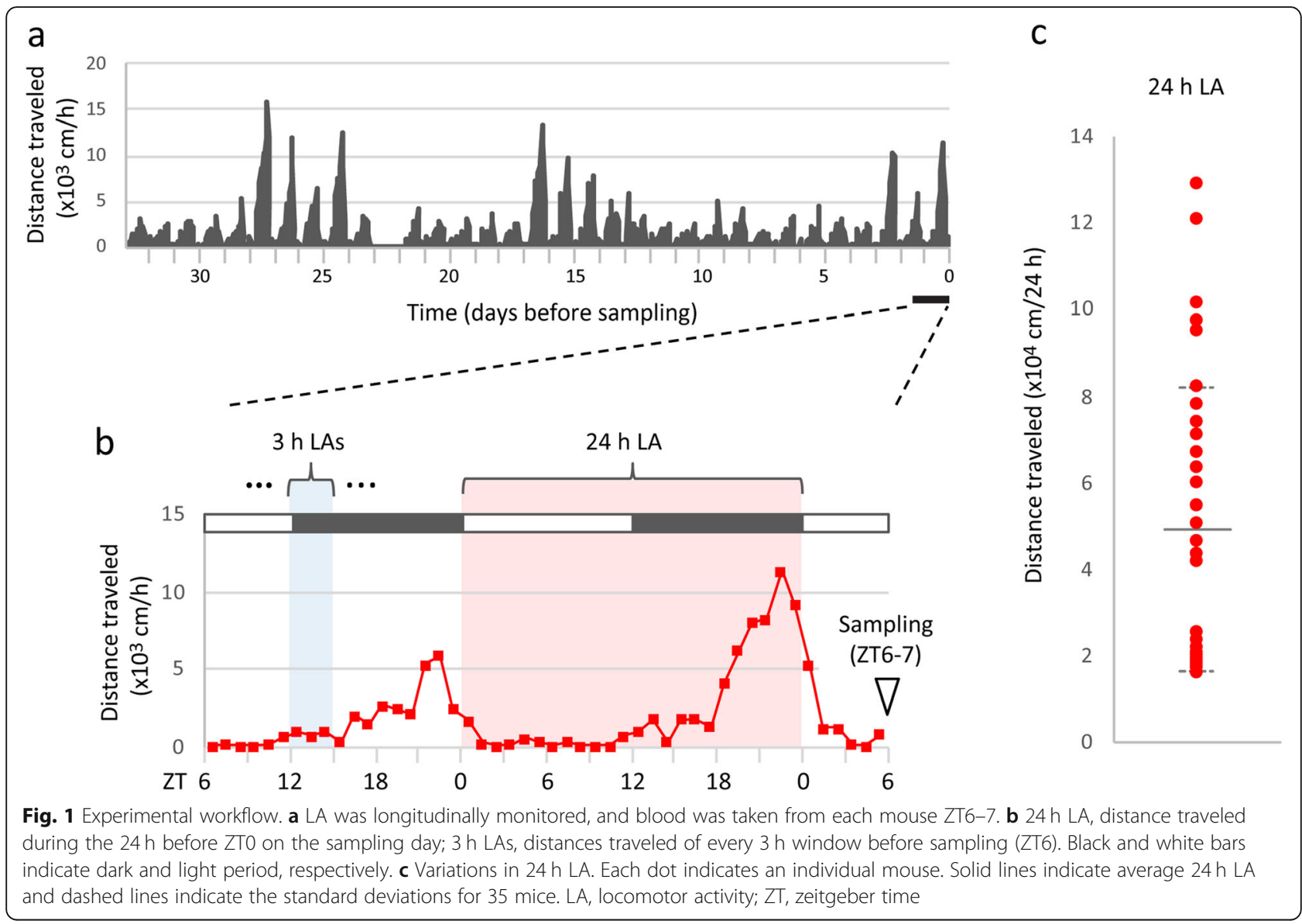

\section{Brain gene expression data}

Expression microarray data of the hippocampal dentate gyrus [29] was obtained from the 35 mice mentioned above. We used data that has been deposited at the Gene Expression Omnibus (GEO; accession number GSE68293) [14]. The $\log _{2}$-transformed expression values were used for the prediction analyses.

\section{Construction of models to predict LA}

The linear regression algorithm was used to predict LA (24 h LA and $3 \mathrm{~h}$ LAs; Fig. 1) and gene expression levels of individual mice from peripheral blood metabolome patterns using MATLAB [14]. The prediction accuracy was evaluated by the leave-one-out cross-validation method and the feature selection for the prediction was conducted by the nested crossvalidation method. Calculation details are included in the Additional file 1: Supplementary Materials and Methods.

\section{Pathway enrichment analysis}

To determine the characteristics of metabolites of interest, we used the default settings in MetaboAnalyst (version 4.0; http://www.metaboanalyst.ca/) [30], a comprehensive, web-based tool for metabolomics analysis and interpretation. Some feature names that had not been recognized in the query were modified if necessary, as suggested in the instructions.

\section{Results}

Locomotor activity can be predicted from blood metabolomic profiles

Of the 106 metabolites tested, the concentrations of 16 features were correlated with $24 \mathrm{~h}$ LA $(P<0.05$, Pearson's correlation; Additional file 1: Figure S1). None of these survived false discovery rate correction for multiple tests (FDR; $q$ value $<0.1$ ). To determine whether the metabolome patterns in peripheral blood could retrospectively predict the $24 \mathrm{~h}$ LA of individual mice, we performed linear regression analysis with the nested cross-validation method [14]. Independent sets of mice were used for feature selection from entire set of 106 metabolites and model fitting in the inner crossvalidation loop and testing the model performance in the outer cross-validation loop. Statistical evaluation of the prediction accuracy of the model revealed a significant correlation between the actual and the predicted $24 \mathrm{~h}$ LA (Fig. 2a), indicating that the metabolome 


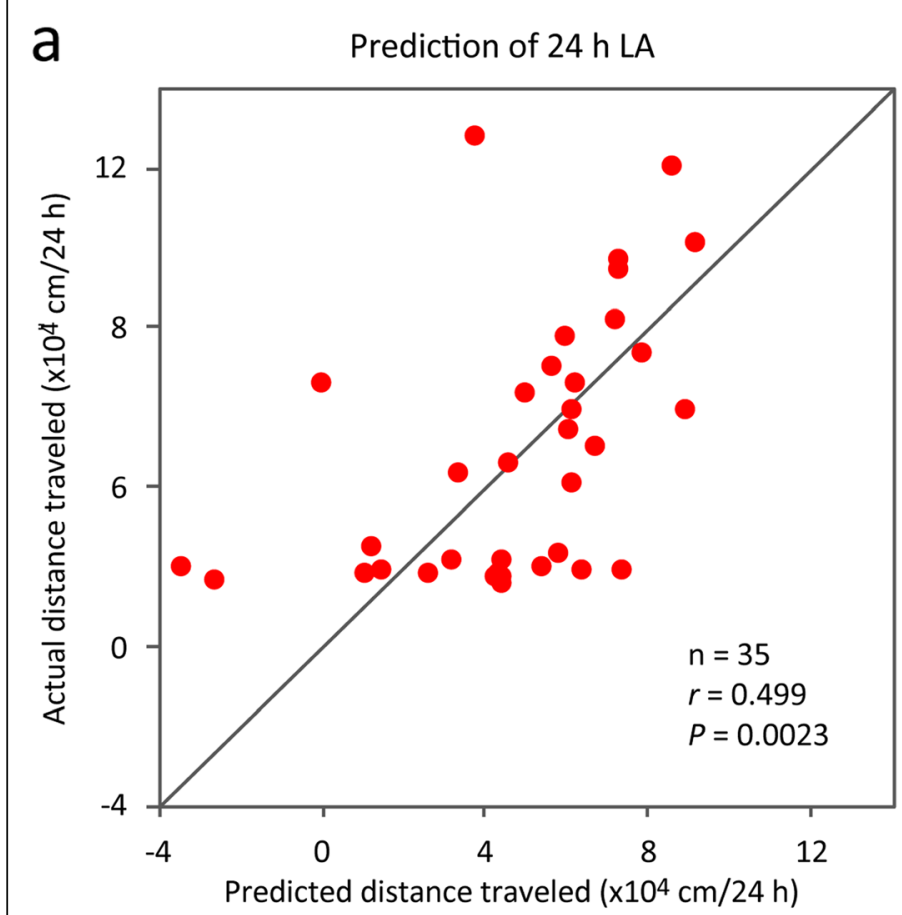

C Term

Warburg Effect

Citric Acid Cycle

Transfer Of Acetyl Groups Into Mitochondria

Carbamoyl Phosphate Synthetase Deficiency

Citrullinemia Type I

Urea Cycle

Gluconeogenesis

Glycogen Storage Disease Type 1A Or Von Gierke Disease

Galactosemia

Galactose Metabolism

b
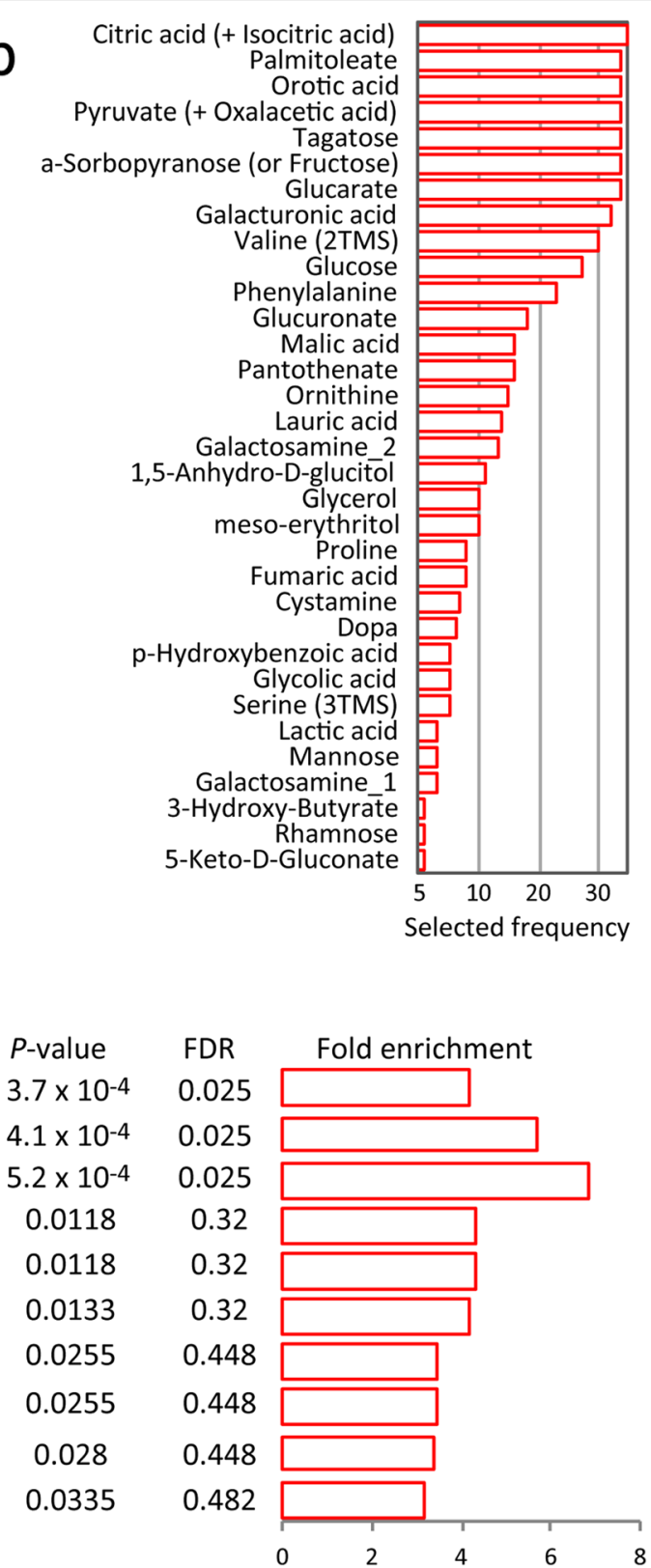

Fig. 2 Metabolome patterns in the peripheral blood can predict $24 \mathrm{~h} \mathrm{LA}$ in Camk2a ${ }^{+/-}$mice. a Scatter plot showing a significant correlation between predicted and actual $24 \mathrm{~h} L A(N=35$ mice). b Feature preference for constructing the $24 \mathrm{~h}$ LA prediction model. c Results of pathway enrichment analysis for the metabolites used for constructing $24 \mathrm{~h} \mathrm{LA}$ prediction model. The statistically enriched terms with raw $P$-value below 0.05 are shown. LA, locomotor activity

patterns in the peripheral blood quantitatively predict the $24 \mathrm{~h}$ LA of individual mice. Thirty-three out of the 106 metabolites were selected to build the successful prediction model (Fig. 2b, Additional file 1: Figure S1).

\section{Mitochondrial alterations implicated in infradian oscillatory LA}

The prediction algorithm we used identified metabolome signatures related to infradian oscillatory LA by weighting metabolites according to their individual predictive strength (Fig. 2b). Next, we examined the weighted metabolites that were selected to predict the $24 \mathrm{~h}$ LA to gain insight into metabolic alterations related to changes in infradian oscillatory LA. Pathway enrichment analysis using a bioinformatics tool MetboAnalyst [30] revealed that the set of metabolites exhibited enrichments in the Warburg effect, the citric acid cycle, and mitochondria-related pathway. These survived multiple comparison correction (FDR $q$ value $<0.05$; Fig. 2c). Interestingly, all the terms were to 
mitochondrial functions (see Discussion), suggesting that mitochondrial function may change concomitantly with infradian oscillatory LA.

\section{Peripheral blood metabolome predicts LA levels for longer time periods}

To investigate whether metabolome patterns in peripheral blood could predict the LA of the past several days, we constructed models for predicting the LA within the 3 days before sampling using a sliding time window (window size: $3 \mathrm{~h}$, step size: $3 \mathrm{~h}$; yielding 24 time windows) ( $3 \mathrm{~h} \mathrm{LAs;} \mathrm{Fig.} \mathrm{1b).} \mathrm{The} \mathrm{actual} \mathrm{and} \mathrm{the} \mathrm{predicted} 3$ $\mathrm{h}$ LAs were similar within the past 3 days in most mice, while differences between them were apparent in some mice (Fig. 3a, Additional file 1: Figure S2). Overall, statistical evaluation of prediction accuracy of the models detected significant correlations between the actual and the predicted $3 \mathrm{~h}$ LAs in 5 of the 24 time windows after FDR correction (time windows of 6-9 h: $r=0.46, P=$ $0.0049 ; 9-12 \mathrm{~h}: r=0.49, P=0.0025 ; 21-24 \mathrm{~h}: r=0.41$, $P=0.016 ; 51-54$ h: $r=0.41, P=0.016 ; 63-66$ h: $r=0.51$, $P=0.0017$; Fig. $3 \mathrm{~b}-\mathrm{g})$. The oldest time window with a significant correlation was from 63 to $66 \mathrm{~h}$ before sampling. These results suggest that multivariate patterns of the metabolome in the peripheral blood hold information about LA of at least the past 3 days. Out of the five time windows mentioned above, metabolites selected were similar in $3 \mathrm{~h}$ LA predictions of 6-9 h and 9-12 h time windows (as well as the $24 \mathrm{~h}$ LA prediction), and differed in other $3 \mathrm{~h}$ time windows (Additional file 1: Figure S3). Metabolites selected for the prediction of the oldest $3 \mathrm{~h}$ time window (63-66 h LA) were associated with aspartate-related pathways (unadjusted $P<0.05$; Additional file 1: Figure S4), which differed from those for $24 \mathrm{~h}$ LA prediction (Fig. 2c).

\section{Hippocampal expression levels of the Arntl gene can be predicted from peripheral blood metabolome}

The regulation of mood is thought to involve the hippocampus [31, 32] and hippocampal expression of some circadian genes are closely related to infradian oscillation of LA in Camk2a $a^{+-}$mice [14]. Consequently, using a prediction approach, we asked whether metabolome patterns in peripheral blood are related to hippocampal expression of circadian genes in relation to mood-changeassociated activity. We used microarray-based gene expression data in the hippocampus of Camk $2 a^{+/-}$mice that we had previously obtained from the same mice whose blood metabolome data was analyzed above [14]. Cross-validated prediction models based on the blood metabolome data were constructed to predict expression levels of genes, focusing on seven circadian-related genes (Lonrf1, Cys1, Hist1h1c, Tef, Ak4, Arntl, and Sfpq), which were among the genes that were selected to predict the 24h LA of Camk2a $a^{+/-}$mice [14]. Of the seven genes tested, Arntl and Sfpq expression levels could be predicted by blood metabolome patterns, which was determined by correlations between actual and predicted expression levels $(P<0.05)$, and Arntl survived correction for multiple testing (Fig. 4a, b). Metabolites used for the successful prediction of hippocampal Arntl expression levels showed enrichment for mitochondria-related pathways (Fig. 4c, d).

\section{Discussion}

In this study, we demonstrated for the first time that peripheral blood metabolomics in combination with a statistical learning algorithm can predict individual levels of mood-change-related activity using an animal model showing exaggerated infradian rhythm. It could be suggested that this result is simply a consequence of the metabolite secretions affected by LA immediately before sampling. However, considering that blood metabolome patterns could not predict LA during the $0-6 \mathrm{~h}$ immediately before sampling (Fig. 3b), this cannot be considered a major factor in determining the blood metabolome patterns. Thus, blood metabolome patterns may hold information about infradian states of LA oscillation during specific time windows that are several hours or days apart from the sampling time.

Our prediction approach highlighted potential alterations in mitochondria metabolism concomitant with infradian mood change-related activity. The Warburg effect is a shift in metabolism towards aerobic glycolysis away from oxidative phosphorylation even in the presence of oxygen which is induced through mitochondrial dysfunction. It was first described in cancer, and studies of both patients' brains and of mouse models have recently been suggested that it could be involved in several psychiatric disorders [24, 33]. Alterations in metabolites related to the citric acid cycle have been found in peripheral blood and cerebrospinal fluid of patients with bipolar disorder [11, 34]. While these studies detected trait-related alterations and support the mitochondria hypothesis of bipolar disorder [35-38], our present study suggests that mitochondrial alterations are related to state-related alterations, or changes in mood states. However, the causal relationship between the mitochondriarelated metabolic alterations in the blood and changes in LA levels remains unclear. Regarding state-related alterations, a recent meta-analysis indicated that blood levels of some inflammatory cytokines, such as IL-6 and sIL-6R, were increased in manic and euthymic states but not the depressive state compared to controls [39]. Other study has shown that blood levels of IL-6 and IL- 2 are positively correlated with mood symptoms, as indicated by the Young Mania Rating Scale [40]. These findings suggest that inflammatory conditions could alter depending on affective states in bipolar disorder, with an enhanced inflammatory- 


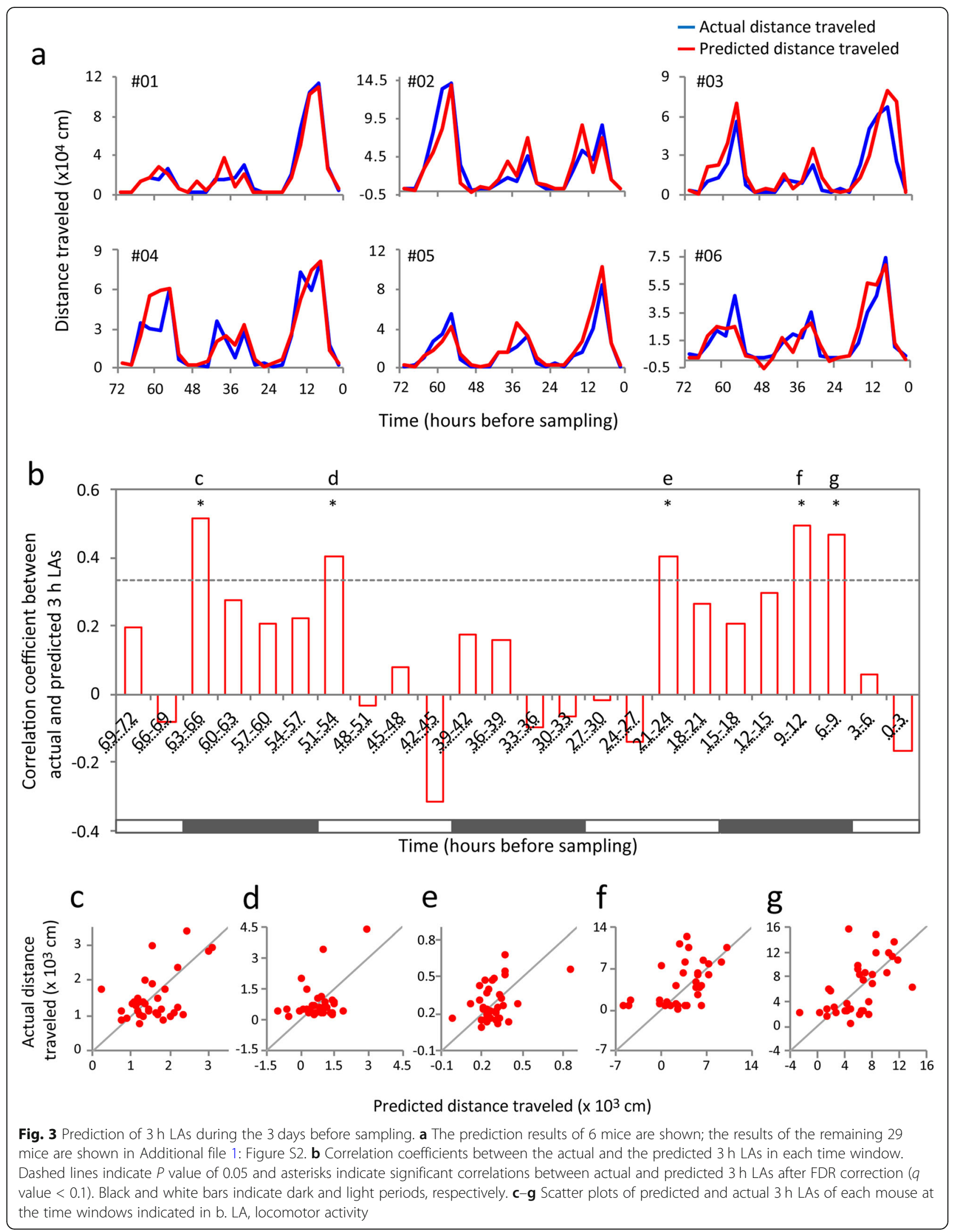




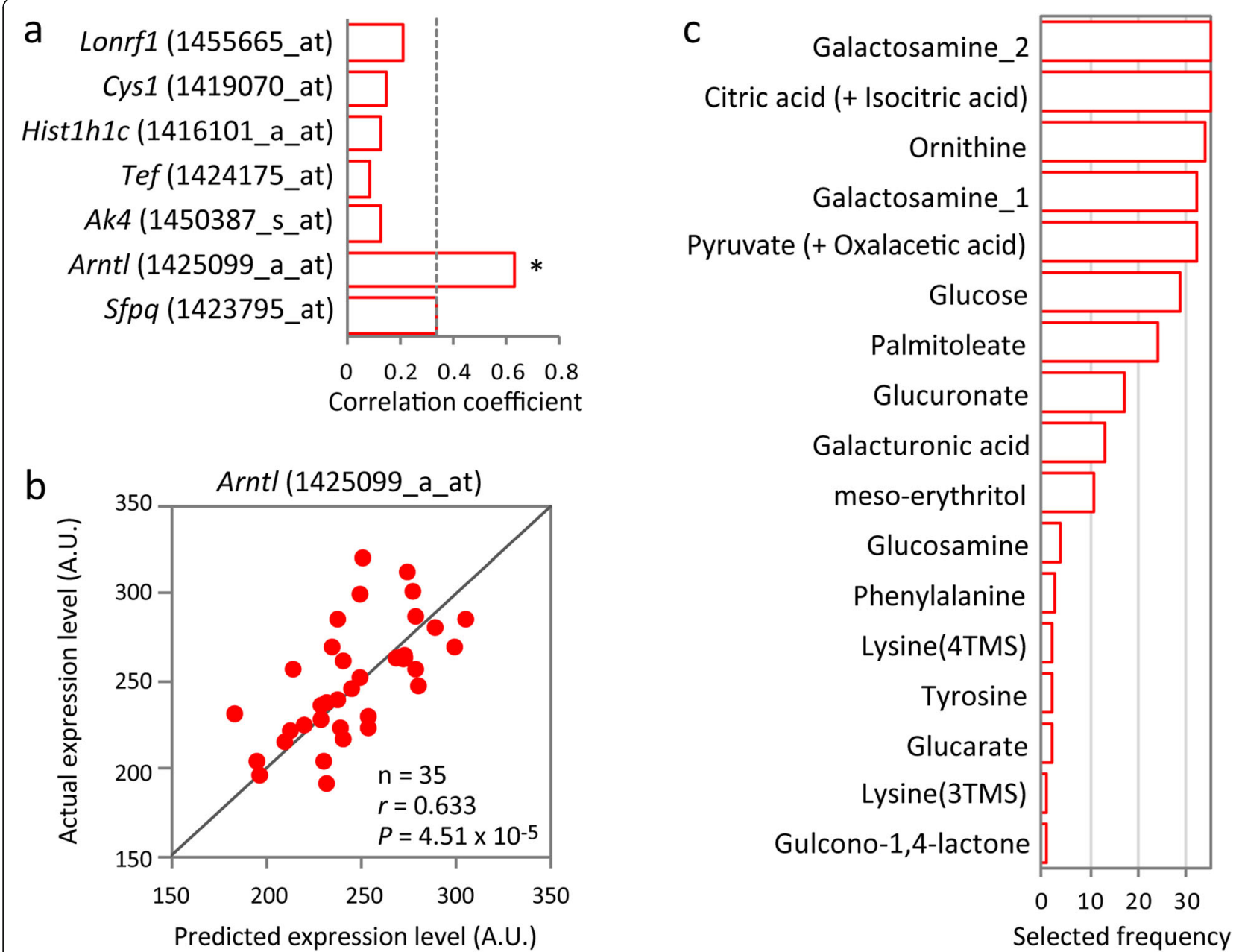

d Term
Transfer Of Acetyl Groups Into Mitochondria
Citric Acid Cycle
Warburg Effect
Urea Cycle
Gluconeogenesis
Glucose-Alanine Cycle
Alanine Metabolism

$P$-value FDR Fold enrichment

$3.2 \times 10^{-4} \quad 0.031$

$0.0014 \quad 0.059$

$0.0018 \quad 0.059$

$0.011 \quad 0.261$

$0.018 \quad 0.297$

$0.018 \quad 0.297$

0.031

0.427

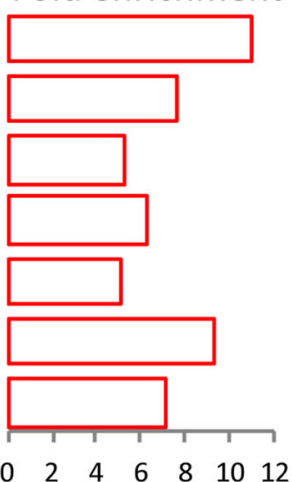

Fig. 4 Prediction of hippocampal gene expression from peripheral blood metabolome. a Genes processed for this prediction analysis were provided from a previous study by Hagihara et al. [14]. The correlation coefficients between actual and predicted expression levels of each gene are shown. Dashed lines indicatea a $P$-value of 0.05 and asterisks indicates significant correlations after Bonferroni correction for multiple testing (significance level: $P<0.00714=0.05 / 7$ ). b Scatter plot showing significant correlations between actual and predicted expression level of Arntl. The correlation was not due to outliers. c Feature preference for constructing the prediction model of Arntl expression level. d Results of pathway enrichment analysis for the metabolites used for constructing the prediction model of Arntl expression level. The statistical enriched terms with raw $P$-value below 0.05 are shown 
related signature in manic states. Supporting these observations, we found that pyruvate, a metabolite suggested to be an endogenous anti-inflammatory molecule [41], was negatively correlated with LA in Camk2a ${ }^{+/-}$mice, and relatively low in manic-like states (Additional file 1: Figure S1). Although increased blood levels of pyruvate has implicated mitochondrial dysfunction in bipolar disorder, its significance on the pathogenesis of the disorder has remained unclear [11]. Considering the findings of the present study and those of studies discussed above, mitochondrial destabilization may be intricately associated with pro- and anti-inflammatory balance in relation to changes in mood states as well as to the pathogenesis of the disorder [42].

We found that blood metabolome patterns predicted $3 \mathrm{~h}$ LA at 3 days before sampling (63-66 h LA; Fig. 3b, c), and metabolites selected for the prediction showed enrichments in aspartate-related pathways (Additional file 1: Figure S4). Alterations in aspartate metabolism have been suggested in the brain and blood of patients with bipolar disorder [11, 43]; however, their relations with mood changes are unknown. Considering that metabolites selected for the prediction of $3 \mathrm{~h} \mathrm{LA}$ at $63-66 \mathrm{~h}$ before sampling and the related metabolic pathways were different from those for $24 \mathrm{~h}$ LA prediction (Fig. 2, Additional file 2: Table S1), levels of a distinct set of blood metabolites and the metabolic pathways might retain information about LA during different and specific time windows.

We also found that the peripheral blood metabolome can predict expression levels of the Arntl gene in the hippocampus, a brain region associated with mood regulation [31, 32], and that the metabolites that successfully predicted these levels showed enrichment for mitochondria-related pathways. These results suggest that systemic alterations in mitochondrial metabolism and hippocampal expression of Arntl, a candidate gene for bipolar disorder [44-46], may serve as a link for brain-periphery interactions in relation to mood-change-associated locomotor activity. Blood metabolites that change according to infradian cyclic locomotor activity could be associated not merely with the physical activity levels, but also potentially with hippocampal function in mood regulation via concomitant expression changes of the bipolar disorder candidate gene.

In conclusion, the present study provided evidence that metabolome patterns in peripheral blood could be used as predictors of states of mood-change-related activity in this mouse model. As a limitation of this study, a recent history of physical activity might change peripheral metabolome patterns independent of the mood state, as intense or moderate exercise might affect mitochondria-related pathways in the brain, heart, and other organs in mice $[47,48]$. Among Camk $2 a^{+/-}$mice, we previously showed that levels of infradian oscillatory LA in the home cages were associated with anxiety-like and depression-like behaviors [14]. Although it is most likely that the LA state in the home cage reflects a certain state of mood in Camk2a $a^{+/-}$mice, the effects of physical activity independent of the mood state on blood metabolome patterns should be considered as potential confounding factors in this study. This study, although limited to an animal model, may have potential for translational applications to human studies, as peripheral blood can be collected from living subjects in a minimally invasive way. States of mood and the related behaviors in human, especially patients with mood disorders, could potentially be retrospectively and prospectively predicted with blood samples through methods analogous to those used here. This may help to develop novel biologically-based methods for the diagnosis and treatment of bipolar disorder. To this end, further studies are needed to determine the most appropriate samples to collect (e.g., blood, saliva, urine, or skin), the appropriate processing methods (e.g., metabolomics, proteomics, or transcriptomics) and to optimize the prediction algorithm [49].

\section{Supplementary information}

Supplementary information accompanies this paper at https://doi.org/10. 1186/s13041-019-0527-3.

\section{Additional file 1. Supplementary Materials and Methods. Figure S1. Correlations between $24 \mathrm{~h} \mathrm{LA}$ and levels of metabolites that were used for the prediction of $24 \mathrm{~h}$ LA. Figure S2. Prediction results of $3 \mathrm{~h}$ LAs from metabolome patterns in peripheral blood of Camk $2 \mathrm{a}^{+/-}$mice. Prediction of $3 \mathrm{~h}$ LAs during the 3 days before sampling for 29 mice. LA locomotor activity. Figure S3. Metabolites used in prediction models that significantly predicted $3 \mathrm{~h} \mathrm{LA}$. Hierarchical clustering of metabolites based on their frequencies selected to build cross-validated prediction models for each time window of LA using R software (http://www.r-project.org/). LA, locomotor activity. Figure S4. Pathway enrichment analysis of the metabolites used for constructing a prediction model of $3 \mathrm{~h}$ LA at 63-66 $\mathrm{h}$ before sampling. The statistically enriched terms with raw $P$-values below 0.05 are shown.}

Additional file 2: Table S1. The raw data used for this study.

\section{Abbreviations}

Ak4: Adenylate kinase 4; Arntl: Aryl hydrocarbon receptor nuclear translocator-like; Camk2a: alpha-isoform of calcium/calmodulin-dependent protein kinase II; Cys1: Cystin 1; FDR: False discovery rate; GC/MS: Gas chromatography mass spectrometry; Hist1h1c: Histone cluster 1, H1c; IL6: Interleukin-6; LA: Locomotor activity; Lonrf1: LON peptidase N-terminal domain and ring finger 1 ; Sfpq: Splicing factor proline/glutamine rich; sIL6R: soluble interleukin-6 receptor; Tef: Thyrotroph embryonic factor; ZT: Zeitgeber time

\section{Acknowledgements}

The authors thank members of the Miyakawa lab for their assistance in animal husbandry.

\section{Authors' contributions}

$\mathrm{HH}, \mathrm{HKN}, \mathrm{JU}$ and $\mathrm{HS}$ performed behavioral experiments and sampling of blood. YI and MY performed GC/MS analysis. TH, HKN and YK performed prediction analyses. $\mathrm{HH}, \mathrm{TH}, \mathrm{HKN}, \mathrm{YI}, \mathrm{HS}, \mathrm{YK}$ and TM. wrote and edited the manuscript. $\mathrm{HH}$. and TM designed the study. All authors read and approved the final manuscript. 


\section{Funding}

This work was supported by JSPS Grant-in-Aid for Scientific Research on Innovative Areas Grant Number JP25116526, JP15H01297, and JP16H06462, JSPS KAKENHI Grant Number JP25242078, and JP15H05710, and AMED Strategic Research Program for Brain Sciences Grant Number JP18dm0107101.

\section{Availability of data and materials}

We provided the raw data used for this study in Additional file 2: Table S1.

\section{Ethics approval and consent to participate}

All animal experiments were approved by the Institutional Animal Care and Use Committee of Fujita Health University, based on the Law for the Humane Treatment and Management of Animals and the Standards Relating to the Care and Management of Laboratory Animals and Relief of Pain.

\section{Consent for publication}

Not applicable.

\section{Competing interests}

The authors declare that they have no competing interests.

\section{Author details}

${ }^{1}$ Division of Systems Medical Science, Institute for Comprehensive Medical Science, Fujita Health University, Kutsukake-cho, Toyoake, Aichi 470-1192, Japan. ${ }^{2}$ Department of Neuroinformatics, ATR Computational Neuroscience Laboratories, Kyoto 619-0288, Japan. ${ }^{3}$ Division of Evidence-based Laboratory Medicine, Kobe University, Graduate School of Medicine, Kobe 650-0017, Japan. ${ }^{4}$ Division of Metabolomics Research, Department of Internal Related, Kobe University Graduate School of Medicine, Kobe 650-0017, Japan. ${ }^{5}$ Division of Gastroenterology, Department of Internal Medicine, Kobe University Graduate School of Medicine, Kobe 650-0017, Japan. ${ }^{6}$ Graduate School of Informatics, Kyoto University, Kyoto 606-8501, Japan.

Received: 24 August 2019 Accepted: 26 November 2019 Published online: 10 December 2019

\section{References}

1. Bryson RW, Martin DF. 17-Ketosteroid excretion in a case of manicdepressive psychosis. Lancet. 1954;267:365-7.

2. Eastwood MR, Whitton JL, Kramer PM, Peter AM. Infradian rhythms. A comparison of affective disorders and normal persons. Arch Gen Psychiatry. 1985;42:295-9.

3. Belmaker RH. Bipolar Disorder. N Engl J Med. 2004;351:476-86.

4. Geddes JR, Miklowitz DJ. Treatment of bipolar disorder. Lancet. 2013;381: 1672-82.

5. Kleine-Budde K, Touil E, Moock J, Bramesfeld A, Kawohl W, Rössler W. Cost of illness for bipolar disorder: a systematic review of the economic burden Bipolar Disord. 2014;16:337-53.

6. Valenza G, Nardelli M, Lanata A, Gentili C, Bertschy G, Kosel M, et al. Predicting mood changes in bipolar disorder through heartbeat nonlinear dynamics. IEEE J Biomed Health Inform. 2016;20:1034-43.

7. Pan Z, Gui C, Zhang J, Zhu J, Cui D. Detecting manic state of bipolar disorder based on support vector machine and gaussian mixture model using spontaneous speech. Psychiatry Investig. 2018;15:695-700.

8. Chen C-H, Suckling J, Lennox BR, Ooi C, Bullmore ET. A quantitative metaanalysis of fMRI studies in bipolar disorder. Bipolar Disord. 2011;13:1-15.

9. Guest PC, Guest FL, Martins-de SD. Making sense of blood-based proteomics and metabolomics in psychiatric research. Int J Neuropsychopharmacol. 2016;19:1.

10. Fernie AR, Trethewey RN, Krotzky AJ, Willmitzer L. Metabolite profiling: from diagnostics to systems biology. Nat Rev Mol Cell Biol. 2004;5:763-9.

11. Yoshimi N, Futamura T, Kakumoto K, Salehi AM, Sellgren CM, HolménLarsson J, et al. Blood metabolomics analysis identifies abnormalities in the citric acid cycle, urea cycle, and amino acid metabolism in bipolar disorder. BBA Clin. 2016:5:151-8

12. Kaddurah-Daouk R, Krishnan KRR. Metabolomics: a global biochemical approach to the study of central nervous system diseases. Neuropsychopharmacology. 2009;34:173-86.

13. Quinones MP, Kaddurah-Daouk R. Metabolomics tools for identifying biomarkers for neuropsychiatric diseases. Neurobiol Dis. 2009;35:165-76.
14. Hagihara H, Horikawa T, Nakamura HK, Umemori J, Shoji H, Kamitani Y, et al. Circadian gene circuitry predicts hyperactive behavior in a mood disorder mouse model. Cell Rep. 2016;14:2784-96.

15. Yamasaki N, Maekawa M, Kobayashi K, Kajii Y, Maeda J, Soma M, et al. Alpha-CaMKII deficiency causes immature dentate gyrus, a novel candidate endophenotype of psychiatric disorders. Mol Brain. 2008;1:6.

16. Le-Niculescu H, Kurian SM, Yehyawi N, Dike C, Patel SD, Edenberg HJ, et al. Identifying blood biomarkers for mood disorders using convergent functional genomics. Mol Psychiatry. 2009;14:156-74.

17. Ament SA, Szelinger S, Glusman G, Ashworth J, Hou L, Akula N, et al. Rare variants in neuronal excitability genes influence risk for bipolar disorder. Proc Natl Acad Sci. 2015;112:3576-81.

18. Li H, Zhou D-S, Chang H, Wang L, Liu W, Dai S-X, et al. Interactome analyses implicated CAMK2A in the genetic predisposition and pharmacological mechanism of bipolar disorder. J Psychiatr Res. 2019:115:165-75.

19. Xing G, Russell S, Hough C, O'Grady J, Zhang L, Yang S, et al. Decreased prefrontal CaMKII alpha mRNA in bipolar illness. Neuroreport. 2002;13:501-5.

20. Mertens J, Wang Q-W, Kim Y, Yu DX, Pham S, Yang B, et al. Differential responses to lithium in hyperexcitable neurons from patients with bipolar disorder. Nature. 2015;527:95-9.

21. Shin R, Kobayashi K, Hagihara H, Kogan JH, Miyake S, Tajinda K, et al. The immature dentate gyrus represents a shared phenotype of mouse models of epilepsy and psychiatric disease. Bipolar Disord. 2013;15:405-21.

22. Hagihara H, Ohira K, Miyakawa T. Transcriptomic evidence for immaturity induced by antidepressant fluoxetine in the hippocampus and prefrontal cortex. Neuropsychopharmacol Rep. 2019;39:78-89.

23. Walton NM, Zhou Y, Kogan JH, Shin R, Webster M, Gross AK, et al. Detection of an immature dentate gyrus feature in human schizophrenia/bipolar patients. Transl Psychiatry. 2012;2:e135.

24. Hagihara H, Catts VS, Katayama Y, Shoji H, Takagi T, Huang FL, et al. Decreased brain $\mathrm{pH}$ as a shared endophenotype of psychiatric disorders. Neuropsychopharmacology. 2018;43:459.

25. Hagihara H, Takao K, Walton NM, Matsumoto M, Miyakawa T. Immature dentate gyrus: an endophenotype of neuropsychiatric disorders. Neural Plast. 2013;2013:e318596

26. Passos IC, Mwangi B, Cao B, Hamilton JE, Wu M-J, Zhang XY, et al. Identifying a clinical signature of suicidality among patients with mood disorders: a pilot study using a machine learning approach. J Affect Disord. 2016;193:109-16.

27. Hagihara H, Ohira K, Toyama K, Miyakawa T. Expression of the AMPA receptor subunits GluR1 and GluR2 is associated with granule cell maturation in the dentate gyrus. Front Neurosci. 2011;5:100.

28. Yonezawa K, Nishiumii S, Kitamoto-Matsuda J, Fujita T, Morimoto K, Yamashita D, et al. Serum and tissue metabolomics of head and neck cancer. Cancer Genomic Proteomic. 2013;10:233-8.

29. Hagihara H, Toyama K, Yamasaki N, Miyakawa T. Dissection of hippocampal dentate gyrus from adult mouse. J Vis Exp. 2009;33:1543.

30. Chong J, Soufan O, Li C, Caraus I, Li S, Bourque G, et al. MetaboAnalyst 4.0: towards more transparent and integrative metabolomics analysis. Nucleic Acids Res. 2018;46:W486-94.

31. David DJ, Wang J, Samuels BA, Rainer Q, David I, Gardier AM, et al. Implications of the functional integration of adult-born hippocampal neurons in anxiety-depression disorders. Neurosci Rev J Bringing Neurobiol Neurol Psychiatry. 2010;16:578-91.

32. Samuels BA, Hen R. Neurogenesis and affective disorders. Eur J Neurosci. 2011;33:1152-9.

33. Vallée A, Vallée J-N. Warburg effect hypothesis in autism spectrum disorders. Mol Brain. 2018;11:1

34. Yoshimi N, Futamura T, Bergen SE, Iwayama $Y$, Ishima T, Sellgren C, et al. Cerebrospinal fluid metabolomics identifies a key role of isocitrate dehydrogenase in bipolar disorder: evidence in support of mitochondrial dysfunction hypothesis. Mol Psychiatry. 2016;21:1504-10.

35. Kato T, Kato N. Mitochondrial dysfunction in bipolar disorder. Bipolar Disord. 2000;2:180-90.

36. Kato T. Mitochondrial dysfunction and bipolar disorder. Curr Top Behav Neurosci. 2011:5:187-200.

37. Morris G, Walder K, McGee SL, Dean OM, Tye SJ, Maes M, et al. A model of the mitochondrial basis of bipolar disorder. Neurosci Biobehav Rev. 2017;74:1-20.

38. Kato T. Current understanding of bipolar disorder: Toward integration of biological basis and treatment strategies. Psychiatry Clin Neurosci. 2019;73: 526 Epub ahead of print. 
39. Goldsmith DR, Rapaport MH, Miller BJ. A meta-analysis of blood cytokine network alterations in psychiatric patients: comparisons between schizophrenia, bipolar disorder and depression. Mol Psychiatry. 2016;21:1696-709.

40. Brietzke E, Stertz L, Fernandes BS, Kauer-Sant'Anna M, Mascarenhas M, Escosteguy Vargas A, et al. Comparison of cytokine levels in depressed, manic and euthymic patients with bipolar disorderJ Affect Disord. 2009;116:214-7.

41. Das UN. Pyruvate is an endogenous anti-inflammatory and anti-oxidant molecule. Med Sci Monit Int Med J Exp Clin Res. 2006;12:RA79-84.

42. Theoharides TC, Zhang B, Conti P. Decreased mitochondrial function and increased brain inflammation in bipolar disorder and other neuropsychiatric diseases. J Clin Psychopharmacol. 2011;31:685-7.

43. Winsberg ME, Sachs N, Tate DL, Adalsteinsson E, Spielman D, Ketter TA. Decreased dorsolateral prefrontal $\mathrm{N}$-acetyl aspartate in bipolar disorder. Biol Psychiatry. 2000;47:475-81.

44. Mansour HA, Wood J, Logue T, Chowdari KV, Dayal M, Kupfer DJ, et al. Association study of eight circadian genes with bipolar I disorder, schizoaffective disorder and schizophrenia. Genes Brain Behav. 2006:5:150-7.

45. Nievergelt CM, Kripke DF, Barrett TB, Burg E, Remick RA, Sadovnick AD, et al. Suggestive evidence for association of the circadian genes PERIOD3 and ARNTL with bipolar disorder. Am J Med Genet B Neuropsychiatr Genet. 2006;141B:234-41.

46. Shi J, Wittke-Thompson JK, Badner JA, Hattori E, Potash JB, Willour VL, et al. Clock genes may influence bipolar disorder susceptibility and dysfunctional circadian rhythm. Am J Med Genet B Neuropsychiatr Genet. 2008;147B: 1047-55.

47. Aguiar AS, Tuon T, Pinho CA, Silva LA, Andreazza AC, Kapczinski F, et al. Intense exercise induces mitochondrial dysfunction in mice brain. Neurochem Res. 2008:33:51-8.

48. Boveris A, Navarro A. Systemic and mitochondrial adaptive responses to moderate exercise in rodents. Free Radic Biol Med. 2008;44:224-9.

49. Hayashi-Takagi A, Vawter MP, Iwamoto K. Peripheral biomarkers revisited: integrative profiling of peripheral samples for psychiatric research. Biol Psychiatry. 2014;75:920-8.

\section{Publisher's Note}

Springer Nature remains neutral with regard to jurisdictional claims in published maps and institutional affiliations.

Ready to submit your research? Choose BMC and benefit from:

- fast, convenient online submission

- thorough peer review by experienced researchers in your field

- rapid publication on acceptance

- support for research data, including large and complex data types

- gold Open Access which fosters wider collaboration and increased citations

- maximum visibility for your research: over $100 \mathrm{M}$ website views per year

At $\mathrm{BMC}$, research is always in progress.

Learn more biomedcentral.com/submissions 